\title{
Neurolymphomatosis of multifocal peripheral nerve involvement: a case report
}

\author{
Ling Peng ${ }^{1 \wedge}$, Hanjin Yang ${ }^{2}$, Yilei Zhao ${ }^{3}$, Jingsong $\mathrm{He}^{4}$, Justin Stebbing $^{5} \wedge$, Bin Chen ${ }^{6}$ \\ ${ }^{1}$ Department of Respiratory Disease, Zhejiang Provincial People's Hospital, Hangzhou, China; ${ }^{2}$ Department of Pathology, The First Affiliated \\ Hospital, School of Medicine, Zhejiang University, Hangzhou, China; ${ }^{3}$ Department of Radiology, The First Affiliated Hospital, School of Medicine, \\ Zhejiang University, Hangzhou, China; ${ }^{4}$ Department of Hematology, The First Affiliated Hospital, School of Medicine, Zhejiang University, \\ Hangzhou, China; ${ }^{5}$ Division of Cancer, Department of Surgery and Cancer, Imperial College London, London, UK; ${ }^{6}$ Department of Orthopedics, \\ The First Affiliated Hospital, School of Medicine, Zhejiang University, Hangzhou, China \\ Correspondence to: Prof. Bin Chen. Department of Orthopedics, The First Affiliated Hospital, School of Medicine, Zhejiang University, Hangzhou \\ 310003, China. Email: ttbin@zju.edu.cn.
}

\begin{abstract}
The infiltration and invasion of nerve trunks, nerve roots, and cranial nerves by lymphomatous malignant cells is defined as "neurolymphomatosis". It is mainly caused by lymphoma cells directly infiltrating the peripheral nerves, with a low incidence. Neurolymphomatosis is a rare condition of neoplastic endoneurial invasion, which is primary or secondary to non-Hodgkin's lymphoma and leukemia. We describe a case of primary peripheral neurolymphomatosis of multifocal involvement in a 77-year-old male patient. He presented with left lower limb pain and was diagnosed with CD20+ diffuse large B cell lymphoma (DLBCL). Magnetic resonance imaging (MRI), fluorine-18 fluorodeoxyglucose $\left({ }^{18} \mathrm{~F}-\mathrm{FDG}\right)$ positron emission tomography (PET) computed tomography (CT), and nerve biopsy contributed to the diagnosis. Genomic profiling, programmed death ligand-1 (PD-L1) expression and tumor mutational burden (TMB) were also assessed. $C D K N 2 A / C D K N 2 B$ deletions have been identified. PD-L1 expression assessed by 28-8 antibody was $1 \%$ positivity, and TMB of the sample was 11.6 muts/Mb. The patient responded well to rituximab combined with chemotherapy, however, he died after 3 cycles of chemotherapy due to severe lung infection and subsequent complication of respiratory failure. Here we report the clinical, radiological, pathological and molecular findings of the patient affected by multifocal neurolymphomatosis without systemic involvement of other organs.
\end{abstract}

Keywords: Neurolymphomatosis; lymphoma; peripheral nerve; next generation sequencing (NGS); case report

Submitted Aug 13, 2021. Accepted for publication Sep 30, 2021.

doi: 10.21037/apm-21-2256

View this article at: https://dx.doi.org/10.21037/apm-21-2256

\section{Introduction}

Lymphoma involving the nervous system is rare. Primary central nervous system lymphomas account for $1 \%$ of all lymphomas, but peripheral nerve system involvement of lymphomas is rather unusual. Lymphomatous infiltration of nerve trunks, nerve roots, plexi, and cranial nerves is a distinct clinical entity called "neurolymphomatosis". Here we report a case of neurolymphomatosis of diffuse large
B cell lymphoma (DLBCL) involvement without cerebral nerve involvement and systemic metastasis. The patient was admitted with lower limb pain and was initially diagnosed with arteriosclerosis obliterans of lower extremities. During several months of checkup and examinations, he was finally diagnosed with neurolymphomatosis. Gene mutation profiling, programmed death ligand-1 (PD-L1) staining and tumor mutational burden (TMB) assessment were

^ ORCID: Ling Peng, 0000-0002-1359-4982; Justin Stebbing, 0000-0002-1117-6947. 

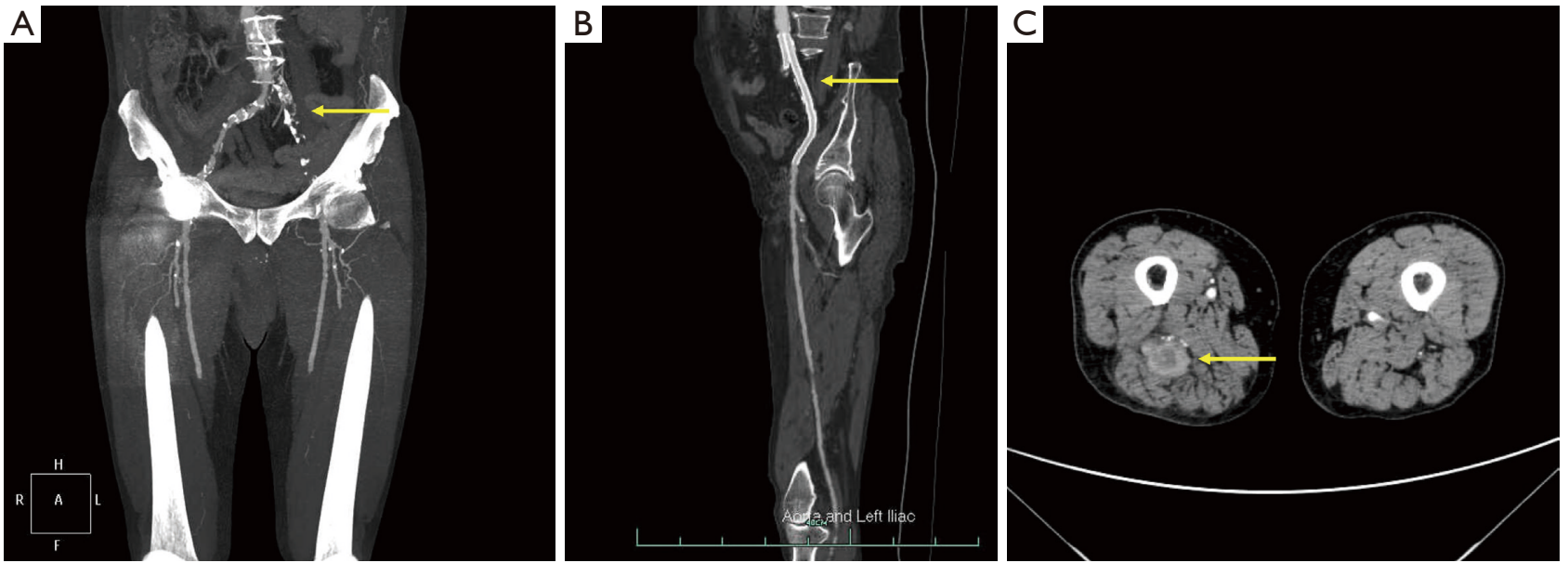

Figure 1 CTA of lower extremity artery. (A) Initial lower extremity artery CTA with arteriosclerosis obliterans of lower extremities (arrow). (B) Re-examination of stent implantation (arrow) at his common and external iliac artery. (C) Axial CT detected a longitudinal enhanced mass in posterior thigh muscles (arrow). This image is published with the consent of the relative of this patient. CTA, computed tomography angiograph; CT, computed tomography.

also performed with the tumor biopsy sample. The patient died of lung infection and respiratory failure after systemic chemotherapy. We present the following case in accordance with the CARE reporting checklist (available at https://apm. amegroups.com/article/view/10.21037/apm-21-2256/rc).

\section{Case presentation}

A 77-year-old male patient presented with pain from his left leg on September 2018. He was admitted to the Department of Vascular Surgery in the First Affiliated Hospital of Zhejiang University. The patient received hip replacement 10 years ago and has been diagnosed with diabetes and coronary heart disease for 7 years. Lower extremity artery computed tomography angiograph (CTA) indicated arteriosclerosis obliterans of lower extremities, with obstruction of the upper segments of his left common, internal and external iliac arteries (Figure 1A). He received stent implantation at his common and external iliac artery. However, the pain worsened after the stent surgery, leading to decreased walking ability.

A second lower extremity artery CTA was performed to check the possible reason on November $22^{\text {nd }}, 2018$. At this time, a longitudinal enhanced mass in posterior thigh muscles was detected (Figure 1B,1C). Magnetic resonance imaging (MRI) study of the thigh revealed masses in right posterior thigh muscle group (Figure 2). In order to further make the diagnosis of the mass, a ${ }^{18}$ fluorodeoxyglucose positron emission tomography computed tomography $\left({ }^{18}\right.$ FDG-PET-CT) was performed, and results confirmed the elevated standard uptake value (SUV) uptake of the suspected lesions and the lymph nodes, extending from the sciatic nerve into the tibial nerve (Figure $3 A$ ). No brain lesions were spotted in PET-CT (Figure 3B). Pelvic cavity MRI discovered multiple lymph nodes alongside the nerve root (Figure 4). Abnormal neurologic findings were decreased vibration sense and decreased ankle reflexes of his right lower limb.

The following laboratory results were normal: routine blood test, erythrocyte sedimentation rate, liver function tests, thyroid function, tumor markers, serologic tests for human immunodeficiency virus (HIV), and EpsteinBarr virus (EBV), hepatitis $\mathrm{B}$ virus (HBV) and hepatitis $\mathrm{C}$ virus (HCV). Blood $\beta 2$ microglobulin level was elevated. Peripheral blood plasma levels of IL-2, IL-6, IL-10 and IL-17A were increased as well. T cell subpopulation test indicated a decrease of fraction of $\mathrm{CD} 3+\mathrm{CD} 8+\mathrm{T}$ cell and $\mathrm{NK}$ cell, and an increase of the fraction of $\mathrm{CD} 3+\mathrm{CD} 4+\mathrm{T}$ helper cell.

He underwent a biopsy for the lesion at sciatic nerve on December $11^{\text {th }}, 2018$. Intraoperative gross examination found that the tumor diffusely expanded the nerve, and epineural dissection was performed to free the mass from the nerve (Figure 5). The intra-operative frozen histology reported a malignant small round cell malignant tumor (MSRCT). The final histological diagnosis was a diffuse 

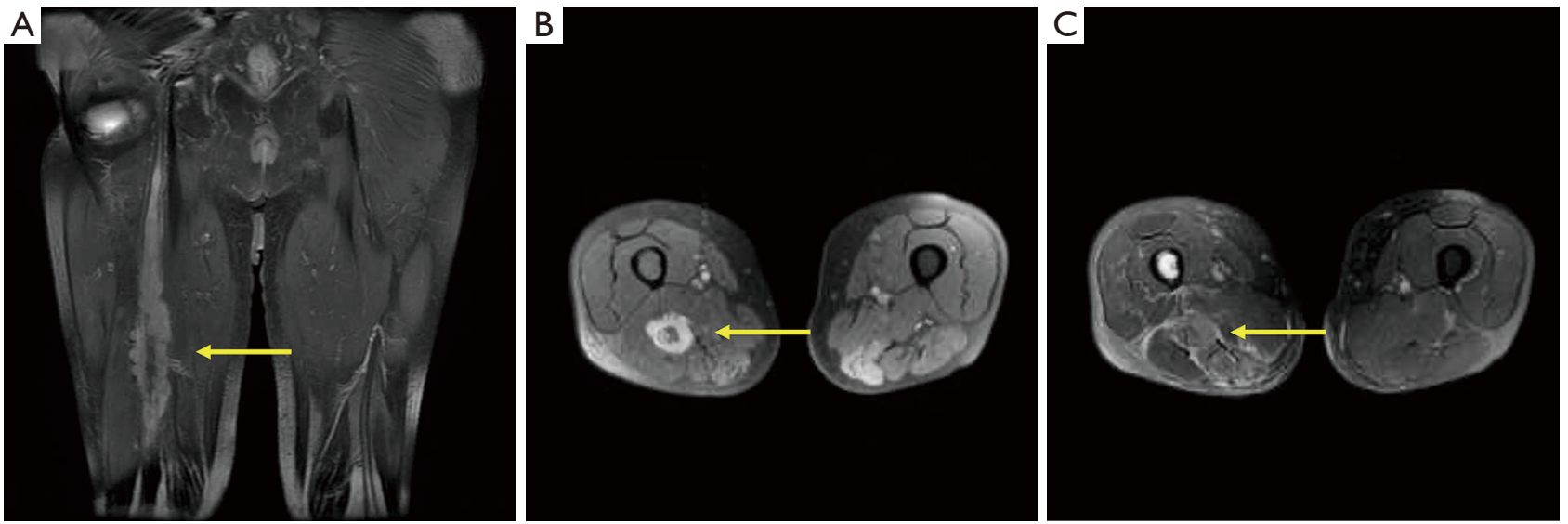

Figure 2 MRI image of thigh. Note the mass (arrows) in muscle in sagittal (A) and axial T1-contrasted (B) and axial T2-weighted (C) MRI sequences. This image is published with the consent of the relative of this patient. MRI, magnetic resonance imaging.

large cell B-cell lymphoma, non-germinal center B cells (GCB), CD3 (-), CD20(+), Ki-67 (+80\%), CD79a(+), CD30(-), CD5(-), CD10(-), Bcl-2(+), Bcl6(+), MUM1 (partial+), PAX-5(+), CD21(-), CyclinD1(-), Myc (+40\%), ALK(-), EBER(-), CD23(-), MPO(-) (Figure 6). PD-L1 expression assessed by $28-8$ antibody was $1 \%$ positivity. Immunohistochemistry (IHC) and fluorescent staining for CD20, S-100, 4',6-diamidino-2-phenylindole (DAPI) and the merged images confirms diffuse positivity of the endoneural infiltrate.

Fresh tumor tissue sections and matched blood from the patient were collected and DNA was extracted from the sample. All coding exons of 466 cancer-related genes and selected introns of 52 common genes rearranged in solid tumors were captured and sequenced. The mean coverage was 1,000Í for tumor tissue and 350Í for blood on Illumina NextSeq platform (Origimed, Inc., Shanghai, China). Genomic alterations including base substitution, short and long insertions/deletions, copy number variations $(\mathrm{CNV})$ and gene rearrangement were determined. Somatic alterations were obtained by comparison with matched blood control samples. The findings from the next generation sequencing (NGS) test were summarized in Table 1. In short, there were 12 tumor-related gene mutations, among which 10 of them were of unknown significance. TMB of the sample was 11.6 muts/Mb, categorized as TMB-H.

A bone marrow biopsy revealed no signs of metastasis. The patient underwent chemotherapy with rituximab $600 \mathrm{mg}$ $\mathrm{d} 0$, cyclophosphomide $600 \mathrm{mg} \mathrm{d} 1$, vinorelbine $20 \mathrm{mg} \mathrm{d} 1$, liposomal doxorubicin $20 \mathrm{mg} \mathrm{d} 1$, and dexamethasone $15 \mathrm{mg}$ d1 -5 , every 3 weeks. The chemotherapy regimen was based on the performance status and age of this patient, in order to minimize toxicity. The patient responded well to the chemotherapy, and his symptoms alleviated 3 days after chemotherapy. Granulocyte colony-stimulating factor (G-CSF) was used but they developed pneumonitis, treated with antibiotics and anti-fungal drugs. However, due to severe lung infection and subsequent complication of respiratory failure, he died after 3 cycles of chemotherapy.

The tests were performed in a centralized clinical testing center. All the methods were performed in accordance with the relevant guidelines and regulations in the First Affiliated Hospital of Zhejiang University and with the Helsinki Declaration (as revised in 2013) (1). The patient was informed, and written consent was collected according to the ethic regulation of the First Affiliated Hospital of Zhejiang University. The relative of the patient has provided informed consent for publication of this case report and accompanying images. A copy of the written consent is available for review by the editorial office of this journal.

\section{Discussion}

We describe a case of neurolymphomatosis of multifocal peripheral nerve involvement with a presentation of recurrent limb pain. Initial imaging and laboratory studies were unremarkable. Clinical staging did not reveal any evidence of systemic involvement of other organs. Neurolymphomatosis is a process of neoplastic endoneurial invasion, mostly associated with non-Hodgkin's lymphoma. When neurolymphomatosis is the first manifestation of 

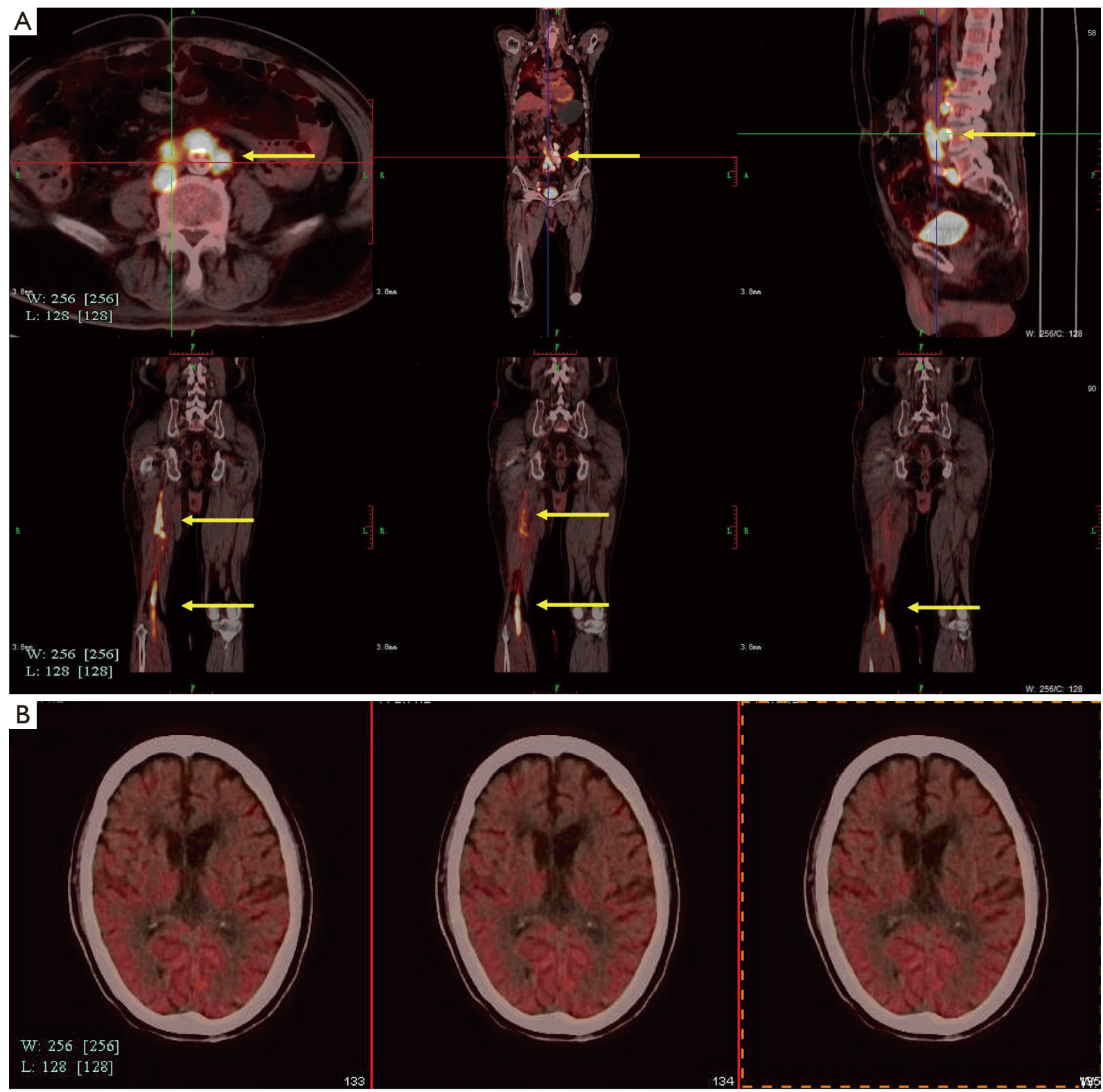

Figure $3{ }^{18}$ FDG-PET image. (A) In the posterior group of the right thigh, there were slightly low-density areas in the middle, along the upper segment of the right sciatic nerve and embryonic nerve, with FDG metabolic enhancement in the right inguinal, horizontal hilar abdominal aorta, right skeletal vessels, pelvic wall and lesions. The arrows indicate lesions of interest. (B) No central nervous system involvement in PET-CT scan. This image is published with the consent of the relative of this patient. ${ }^{18}$ FDG-PET, ${ }^{18}$ fluorodeoxyglucose positron emission tomography; CT, computed tomography.

the hematological malignancy, the situation is defined as primary neurolymphomatosis (2). Neurolymphomatosis is a rare complication of lymphoma and leukemia. Patients can present with primary neurolymphomatosis, but the majority of patients present as secondary neurolymphomatosis, in which neurolymphomatosis is a result of relapse or progression of a previously diagnosed hematologic malignancy (3). There are multiple reasons accounting for peripheral nerve system involvement in lymphoma, including paraneoplastic direct peripheral nerve system infiltration and peripheral nerve system involvement due to chemotherapy and radiotherapy (4). Although the 

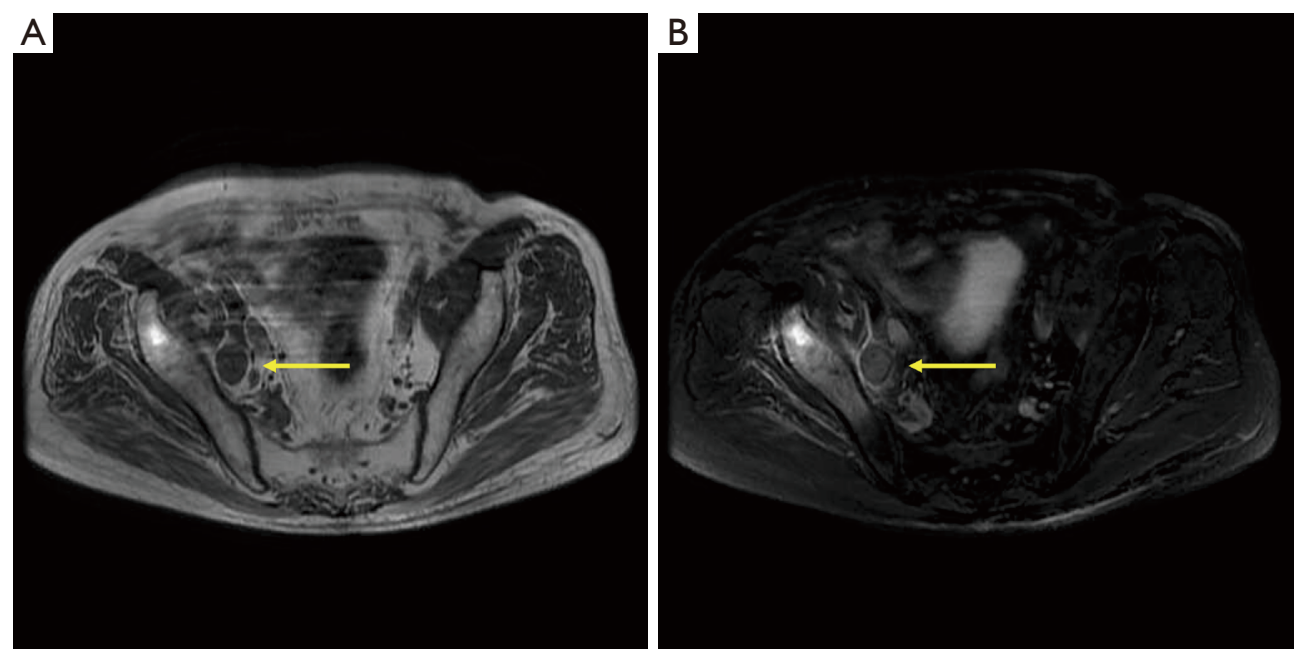

Figure 4 Pelvic MRI image. Pelvic MRI image indicated the lymph node enlargement. (A) Axial T1 and (B) T2-weighted MRI sequences. The arrows indicate metastatic lesion of interest. This image is published with the consent of the relative of this patient. MRI, magnetic resonance imaging.
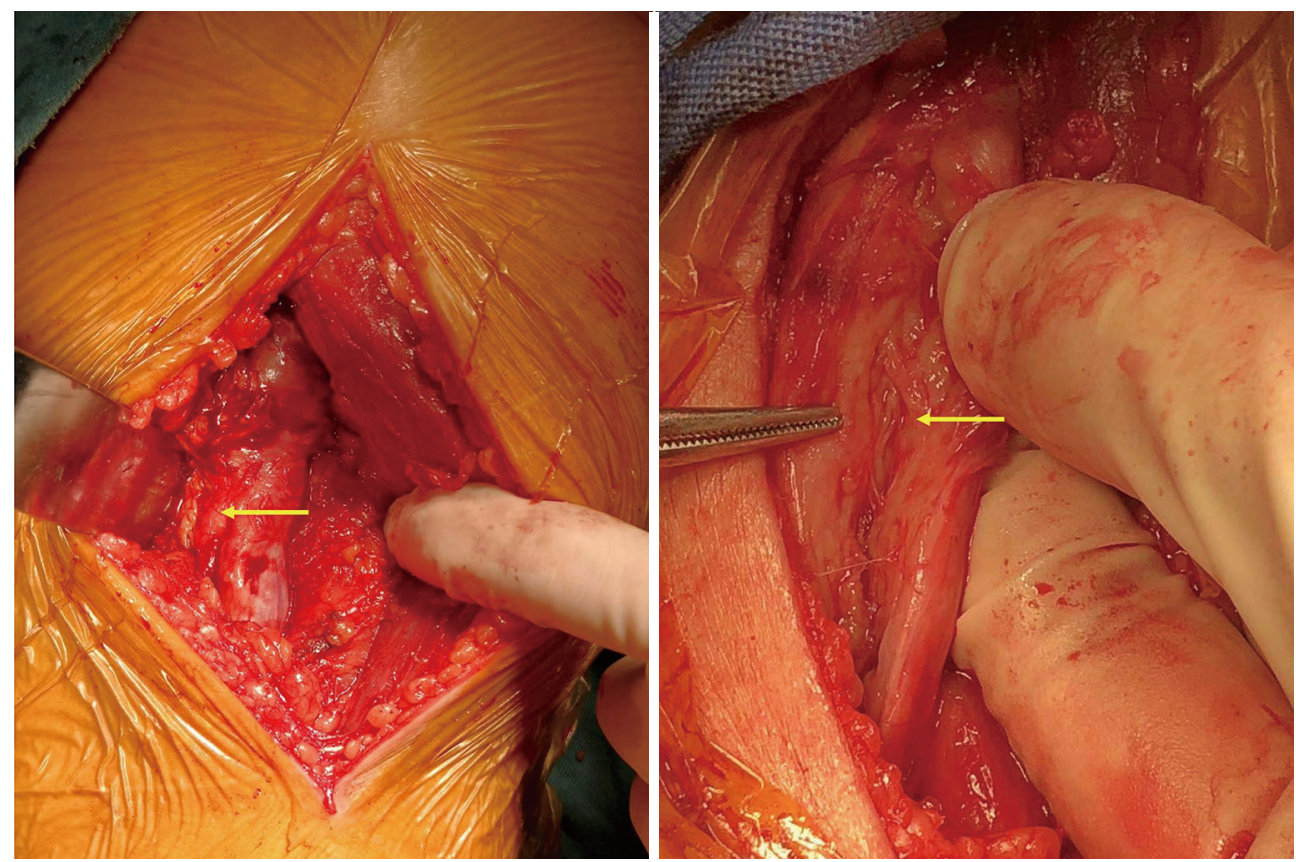

Figure 5 Intraoperative examination of the tumor. Intraoperative examination indicated that the tumor diffusely expanded the nerve (arrows). This image is published with the consent of the relative of this patient.

involvement of peripheral nerves has been previously described, primary involvement of the multifocal peripheral nerves is very rare.

Neurolymphomatosis is frequently misdiagnosed. The general symptoms of lymphoma are fever, night sweats, weight loss, anemia, hepatosplenomegaly and multiple lymphadenopathies, which were not manifested in this patient. History of diabetes and coronary heart disease of this patient and his age would lead the doctors suspect possible reasons of leg pain as lumbar disc 

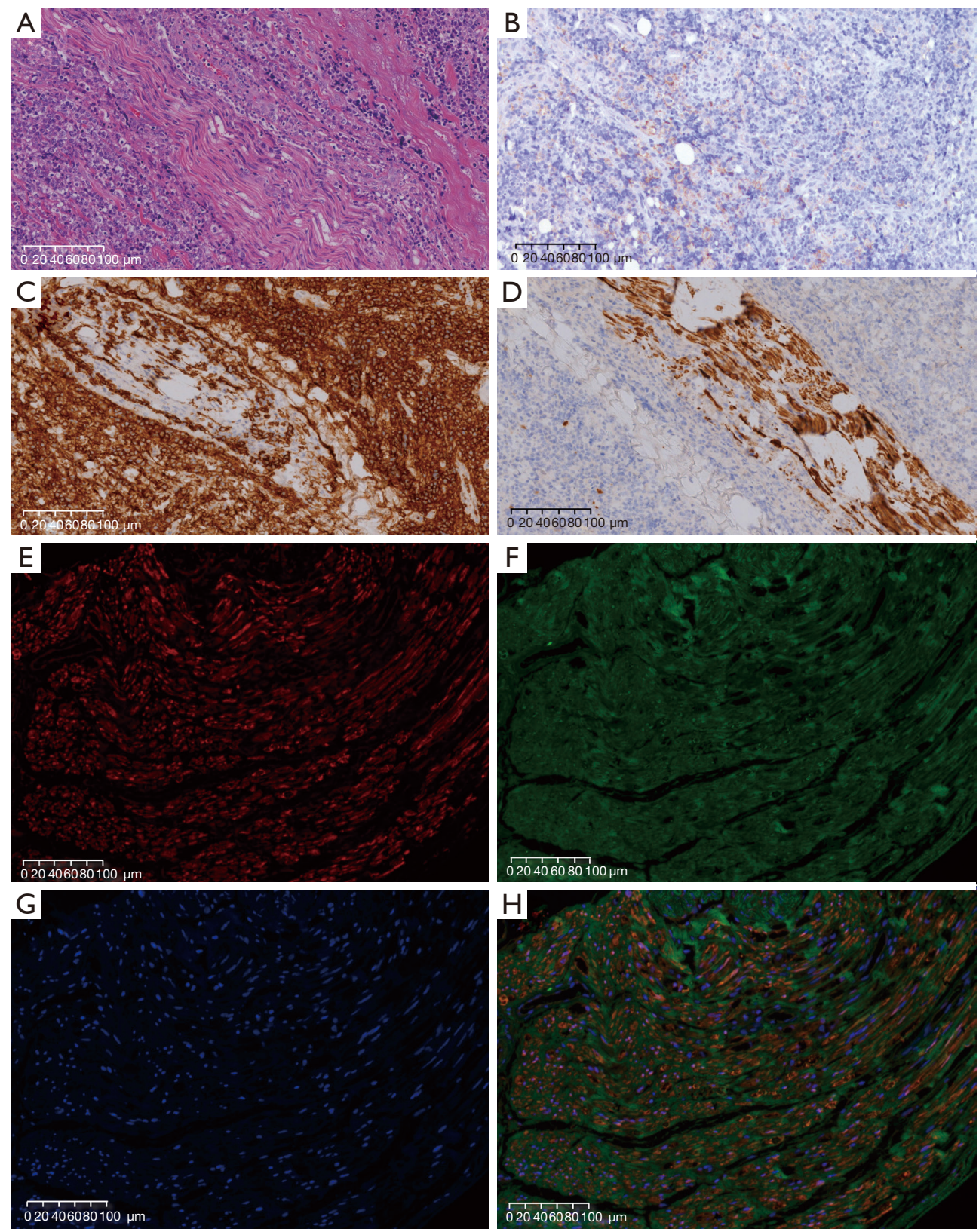

Figure 6 Pathological demonstration of the lymphoma. A gray-white strip with a size of $1.7 \times 0.7 \times 0.3 \mathrm{~cm}$ was sent to the histologist. Tumors invade into nerve tracts. Tumor cells are round and diffusely distributed, with large nuclei and prominent nucleoli. Mitosis is common. Tumors destroy nerve bundles and invade along nerve bundles. There is no invasion of extra-perineurial adipose tissue of nerve bundles. (A) Hematoxylin and erosin staining; original magnification 200×. (B) PD-L1 staining of the tumor. IHC staining image for CD20 (C) and S-100 (D). Tumor cells were diffusely positive for CD20, while the nerve bundles surrounded by tumors were negative. Nerve tracts are positive for S-100, with nerve fibers destroyed, while tumor cells surrounding, and invading nerve fibers are negative. Fluorescent staining for (E) CD20 (red), (F) S-100 (green), and (G) DAPI (blue). (H) Merged image for CD20, S-100, and DAPI. This image is published with the consent of the relative of this patient. IHC, immunohistochemistry; DAPI, 4',6-diamidino-2-phenylindole. 
Table 1 Somatic mutations of the tumor

\begin{tabular}{ll}
\hline Gene & Information \\
\hline CDKN2A & Gene deletion \\
CDKN2B & Gene deletion \\
PMS2 & C.538-2A>G (splicing site change) \\
RET & R114H: exon 3 \\
CD79B & Y197D: exon 5 \\
ETV6 & C.33+1G>A (splicing site change) \\
& R49C: exon 2 \\
KMT2D & KMT2D/PRKAG1 rearrangement \\
LRP1 & A844V: exon 16 \\
MYD88 & P266L: exon 4 \\
RHOA & W58S: exon 3 \\
SPEN & M1714V: exon 11 \\
\hline
\end{tabular}

protrusion, diabetic neuropathy or artery occlusion. While differentiating peripheral neuropathy, a suspicion of neurolymphomatosis is required since presenting symptoms vary, but conventional radiology such as CT scan has only modest sensitivity. MRI and electrophysiological studies would avoid this misinterpretation, as the appearance on MRI is associated with malignancies $(5,6) .{ }^{18} \mathrm{FDG}-$ PET is up to now the most sensitive and specific imaging examination for lymphoma (7). These imaging techniques would help the clinicians make the right diagnosis.

Nerve biopsy can be a valuable diagnostic tool in this setting. The difficulty of diagnosis was also due to the need for a biopsy to confirm the diagnosis, which is often hard to perform since not all of the lesions are accessible during a biopsy. The local sign of lymphomas is usually a round or oval mass, while peripheral nerve lymphoma can present as diffuse or nodular nerve thickening, or as a mass. In this patient the lymphoma grew alongside the nerves, showing a long strip of thickening of the mass (8). Neurolymphomatosis spreads along a dominant nerve toward the spinal canal and moves circumdurally to affect the corresponding contralateral nerve, which provides information to understand the spread of the disease (9).

NGS is currently used in lymphoma patients to characterize the genomic profiling and identify gene mutations. Recent NGS-based studies have offered a comprehensive knowledge of the genetic landscapes featuring lymphomas for better subclassifications (10).
Results from a LYSA study demonstrated the contribution of NGS with a gene panel to personalized treatment in DLBCL and inform prognosis (11). In this patient, we found deletion of $C D K N 2 A$, a tumor suppressor gene encoding $C D K N 2 A$, which is co-located with its homologous gene $C D K N 2 B$ on human chromosome 9p21 (12). CDKN2A and CDKN2B inhibit CCND1/CDK4/6 complex formation, which prevents $\mathrm{RB} 1$ phosphorylation which is necessary to the $\mathrm{G} 1 / \mathrm{S}$ transition. CDKN2 gene is a major tumor suppressor gene located on chromosome $9 \mathrm{p} 21$, and it is involved in the tumorigenesis of lymphoid malignancies (13). Previous studies have shown that $C D K N 2 A / C D K N 2 B$ deletions are frequent in non-Hodgins's lymphomas, which is an adverse prognostic marker for survival $(14,15)$.

Researchers have demonstrated significantly increased numbers of programmed death receptor 1 (PD-1)+ tumorinfiltrating lymphocytes (TILs) in B cell lymphomas (16). PD-L1 expression is a characteristic of a subset of aggressive B-cell lymphomas (17). A meta-analysis indicated that high expression of PD-L1 was a poor prognostic biomarker in patients with NHL (18). TMB has also proven to be correlated with the efficacy of immunotherapy in multiple malignancies, while the cut-off value for lymphoma has not been decided. This report used 10 muts/Mb as a cutoff value due to the result from the phase III clinical trial CheckMate 227 (19). PD-L1 and TMB data provided TMB information for regimen choices, such as immune checkpoint inhibitor, which has become an extremely active area in clinical study of lymphoma $(20,21)$.

Early diagnosis of lymphoma is important in order to initiate local and systemic therapy. In the case of neurolymphomatosis, early detection does not always translate into better prognosis. The prognosis of DLBCL varies greatly according to different origins. It's unclear whether neurolymphomatosis has distinct biological behavior compared with other lymphomas. The prognosis and general outcome of neurolymphomatosis is not yet defined, possibly due to rare reports and limited follow-up period.

There are several highlighted points in our case report. Firstly, this is a rare case of elderly patient whose symptom was initially presented as pain, but finally diagnosed with primary neurolymphomatosis. The case would give more information regarding the avoidance of misdiagnosis when neurolymphomatosis is the first manifestation of lymphoma. Secondly, unique to this case was the initial multifocal peripheral nerve involvement without central nerve or additional systemic involvement. The detailed mechanism 
why the lymphomatous cells would preferentially invade into the peripheral nerve in this patient is unknown. Thirdly, none of the previously reported cases of neurolymphomatosis underwent NGS testing for possible gene mutation sites identification.

In conclusion, we describe a case of neurolymphomatosis with multifocal peripheral nerve involvement. It's a rare presentation of lymphoma but a potentially treatable condition, which might be frequently misdiagnosed. The possibility of neurolymphomatosis should be considered in patients with recurrent pain symptoms.

\section{Acknowledgments}

We thank Prof. Xiaojun Zhou from Nanjing Jinling Hospital for providing helpful insights and suggestions and professional guidance. The authors would like to thank the patient's family for giving consent and for providing the detail information of this case.

Funding: None.

\section{Footnote}

Reporting Checklist: The authors have completed the CARE reporting checklist. Available at https://apm.amegroups. com/article/view/10.21037/apm-21-2256/rc

Conflicts of Interest: All authors have completed the ICMJE uniform disclosure form (available at https://apm. amegroups.com/article/view/10.21037/apm-21-2256/coif). Professor JS has sat on SABs for Vaccitech, Heat Biologics, Eli Lilly, Alveo Technologies, Pear Bio, Agenus, Equilibre Biopharmaceuticals, Graviton Bioscience Corporation, Celltrion, Volvox, Certis Oncology Solutions, Greenmantle, Zedsen, Bryologyx and Benevolent AI. He has consulted with Lansdowne partners and Vitruvian. He sits on the Board of Directors for Xerion and BB Biotech Healthcare Trust PLC. None are relevant here. The other authors have no conflicts of interest to declare.

Ethical Statement: The authors are accountable for all aspects of the work in ensuring that questions related to the accuracy or integrity of any part of the work are appropriately investigated and resolved. The tests were performed in a centralized clinical testing center. All the methods were performed in accordance with the relevant guidelines and regulations in the First Affiliated Hospital of Zhejiang University and with the Helsinki Declaration (as revised in 2013). The patient was informed, and written consent was collected according to the ethic regulation of the First Affiliated Hospital of Zhejiang University. The relative of the patient has provided informed consent for publication of this case report and accompanying images. A copy of the written consent is available for review by the editorial office of this journal.

Open Access Statement: This is an Open Access article distributed in accordance with the Creative Commons Attribution-NonCommercial-NoDerivs 4.0 International License (CC BY-NC-ND 4.0), which permits the noncommercial replication and distribution of the article with the strict proviso that no changes or edits are made and the original work is properly cited (including links to both the formal publication through the relevant DOI and the license). See: https://creativecommons.org/licenses/by-nc-nd/4.0/.

\section{References}

1. World Medical Association. World Medical Association Declaration of Helsinki: ethical principles for medical research involving human subjects. JAMA 2013;310:2191-4.

2. Grisariu S, Avni B, Batchelor TT, et al. Neurolymphomatosis: an International Primary CNS Lymphoma Collaborative Group report. Blood 2010;115:5005-11.

3. Baehring JM, Damek D, Martin EC, et al. Neurolymphomatosis. Neuro Oncol 2003;5:104-15.

4. Shree R, Goyal MK, Modi M, et al. The diagnostic dilemma of neurolymphomatosis. J Clin Neurol 2016;12:274-81.

5. Capek S, Hébert-Blouin MN, Puffer RC, et al. Tumefactive appearance of peripheral nerve involvement in hematologic malignancies: a new imaging association. Skeletal Radiol 2015;44:1001-9.

6. Murthy NK, Hébert-Blouin MN, Capek S, et al. Understanding the pathognomonic tumefactive appearance of neurolymphomatosis: a unifying theory of neurolymphoma. World Neurosurg 2020;141:e490-7.

7. Campagnolo $M$, Cacciavillani $M$, Cavallaro $T$, et al. Neurolymphomatosis, a rare manifestation of peripheral nerve involvement in lymphomas: suggestive features and diagnostic challenges. J Peripher Nerv Syst 2020;25:312-5.

8. Keddie S, Nagendran A, Cox T, et al. Peripheral nerve neurolymphomatosis: Clinical features, treatment, and 
outcomes. Muscle Nerve 2020;62:617-25.

9. Murthy NK, Amrami KK, Spinner RJ. Circumdural extension of perineural spread leading to bilateral disease in neurolymphomatosis. Acta Neurochir (Wien) 2020;162:3197-200.

10. Heimann P, Dewispelaere L. Indications of nextgeneration sequencing in non-Hodgkin's lymphoma. Curr Opin Oncol 2020;32:391-7.

11. Dubois S, Viailly PJ, Mareschal S, et al. Next-generation sequencing in diffuse large B-cell lymphoma highlights molecular divergence and therapeutic opportunities: a LYSA study. Clin Cancer Res 2016;22:2919-28.

12. Kamb A, Gruis NA, Weaver-Feldhaus J, et al. A cell cycle regulator potentially involved in genesis of many tumor types. Science 1994;264:436-40.

13. Uchida T, Kinoshita T, Saito H, et al. CDKN2 (MTS1/p16INK4A) gene alterations in hematological malignancies. Leuk Lymphoma 1997;24:449-61.

14. Alhejaily A, Day AG, Feilotter HE, et al. Inactivation of the CDKN2A tumor-suppressor gene by deletion or methylation is common at diagnosis in follicular lymphoma and associated with poor clinical outcome. Clin Cancer Res 2014;20:1676-86.

15. Braun M, Pastorczak A, Fendler W, et al. Biallelic loss of

Cite this article as: Peng L, Yang H, Zhao Y, He J, Stebbing J, Chen B. Neurolymphomatosis of multifocal peripheral nerve involvement: a case report. Ann Palliat Med 2022;11(7):2529-2537. doi: 10.21037/apm-21-2256
CDKN2A is associated with poor response to treatment in pediatric acute lymphoblastic leukemia. Leuk Lymphoma 2017;58:1162-71.

16. Muenst S, Hoeller S, Willi N, et al. Diagnostic and prognostic utility of PD-1 in B cell lymphomas. Dis Markers 2010;29:47-53.

17. Chen BJ, Chapuy B, Ouyang J, et al. PD-L1 expression is characteristic of a subset of aggressive B-cell lymphomas and virus-associated malignancies. Clin Cancer Res 2013;19:3462-73.

18. Zhao $\mathrm{S}$, Zhang $\mathrm{M}$, Zhang $\mathrm{Y}$, et al. The prognostic value of programmed cell death ligand 1 expression in nonHodgkin lymphoma: a meta-analysis. Cancer Biol Med 2018:15:290-8.

19. Hellmann MD, Ciuleanu TE, Pluzanski A, et al. Nivolumab plus ipilimumab in lung cancer with a high tumor mutational burden. N Engl J Med 2018;378:2093-104.

20. Zhang J, Medeiros LJ, Young KH. Cancer immunotherapy in diffuse large B-cell lymphoma. Front Oncol 2018;8:351.

21. Lin N, Song Y, Zhu J. Immune checkpoint inhibitors in malignant lymphoma: Advances and perspectives. Chin J Cancer Res 2020;32:303-18. 